\title{
IMRT Treatment for Nasopharyngeal Carcinoma (NPC) Is Superior for Relieving Xerostomia, Improving Quality of Life and Protecting the Parotid Glands
}

\section{Linjun Hu ( $\nabla$ hulinjunmrs@163.com )}

The First Hospital of Shanxi Medical University

\section{Xianfeng Li}

The First Hospital of Shanxi Medical University

\section{Zhenzhen Wu}

The First Hospital of Shanxi Medical University

\section{Xiongwei Wang}

The First Hospital of Shanxi Medical University

\section{Ruijuan Meng}

The First Hospital of Shanxi Medical University

\section{Yanlan Zhang}

The First Hospital of Shanxi Medical University

\section{Research}

Keywords: IMRT, NPC, Xerostomia, Quality of life, Parotid gland

Posted Date: January 5th, 2021

DOl: https://doi.org/10.21203/rs.3.rs-137696/v1

License: (c) (1) This work is licensed under a Creative Commons Attribution 4.0 International License.

Read Full License 


\section{Abstract}

Background: We investigated the effects of intensity-modulated radiotherapy (IMRT) on xerostomia, quality of life (QoL) and protection of the parotid glands in patients with nasopharyngeal carcinoma (NPC) compared to three-dimensional conformal radiotherapy (3D-CRT).

Methods: Eighty patients with NPC were enrolled in a prospective randomized clinical study and received IMRT or 3D-CRT. A xerostomia questionnaire (XQ), QoL questionnaire and parotid gland function assessment were completed at baseline and 6 months after radiotherapy.

Results: Xerostomia occurred in both groups after RT, although the XQ scores of the IMRT group were lower and, as a consequence, the QoL scores were higher than in the 3D-CRT group. A correlation analysis showed that there was some correlation between the XQ and QoL scores. After 6 months of RT, the uptake index ( $\mathrm{UI}$ ) and excretion index (EI) decreased by $34.41 \%$ and $22.66 \%$ in the IMRT group, while in the 3D-CRT group the UI and El decreased by $54.4 \%$ and $41.9 \%$, respectively, and the function of the parotid glands declined.

Conclusions: IMRT better relieved xerostomia and improved the QoL of NPC patients by sparing the parotid glands, without compromising treatment of the target site.

\section{Introduction}

Nasopharyngeal carcinoma (NPC) is a common malignant tumor in the head and neck arising from the nasopharynx epithelium. The incidence of NPC exhibits geographical variation with high rates of up to $15 \sim 20$ per 10 million (women) or $20 \sim 30$ per 10 million (men) in South and Southeast Asia(1-3). In terms of demographic trends, the incidence is about 1.5 -fold higher in men than in women in China(4), and the peak age of onset ranges from 50 to 60 years.

Although NPC and other head and neck tumors have similar cell or tissue lineage, NPC has unique biological characteristics. As the nasopharyngeal cavity is deep and narrow, strong local invasion and higher lymph node metastasis rates occur. Besides, it is adjacent to the brainstem, temporal lobe, optic chiasm, cervical spinal cord, temporomandibular joint, parotid gland and other important organs. These characteristics mean that surgical therapy and radical excision are conducted with considerable difficulty.

Morphologically, an abundance of lymphoid cells is often seen intermixed with transformed epithelial cells, but NPC is widely regarded to be squamous in origin. Depending on the degree of differentiation and on the basis of WHO criteria, NPC is categorized into three pathological subtypes. Type I corresponds to differentiated tumors with surface keratin, whereas types II and III are defined as non-keratinizing differentiated and undifferentiated tumors, respectively $(2,3)$. The non-keratinizing subtypes constitute most cases that are highly malignant and sensitive to radiation, which provides favorable conditions for radiotherapy. Therefore, radiotherapy is the most basic and main treatment for NPC. The 5-year survival rate of early NPC after radiotherapy can reach $80 \%(5)$. 
For radiotherapy, the clinical target area of NPC includes the area from the upper part of the base of the skull to the lower edge of the clavicle. Moreover, the shape of the large target area is extremely irregular, and the lymphatic drainage area is extremely complex. Therefore, conventional radiotherapy cannot effectively spare the adjacent important organs and normal tissues, especially the parotid gland, which inevitably receives high-dose radiation, and the other salivary glands, which exhibit tissue fibrosis and even shrinkage after irradiation, leading to irreversible impairment of function. One of the most common and long-term sequelae is xerostomia, which seriously affects the quality of life (QoL) of patients as it impacts speaking, nutrition, taste, sleep and so on. Some patients cannot even complete their radiotherapy plan on time, thus decreasing the local control rate of the tumor. In recent years, more attention has been paid to the QoL of NPC patients, focusing on ways to protect the salivary glands and reduce sequelae after treatment, especially xerostomia(6).

Radiotherapy is the primary and only curative treatment for NPC. In recent years, intensity-modulated radiotherapy (IMRT) has been used widely in the treatment of NPC, as it is a technique that allows minimization of the radiation doses to adjacent critical normal structures, while maintaining or increasing the primary tumor site dose. On the basis of this, IMRT can improve target coverage and spare important tissues when applied to NPC patients. The advantages of IMRT make it the preferred treatment method in NPC, compared to conventional three-dimensional conformal radiotherapy (3D-CRT). This has been confirmed in some previous studies. For example, a study by Kristensen et al. indicated that IMRT improved the target volume coverage and protected critical organs compared with 3D-CRT, which suggested that IMRT is better than 3D-CRT in the treatment of patients with NPC(7). Vergeer et al. reported that IMRT treatment resulted in a significant reduction in the radiation dose of the parotid glands and less xerostomia, as well as other side-effect symptoms, compared to conventional 3D-CRT(8). A study by Pow et al. showed that IMRT was significantly superior to CRT in protecting the salivary glands and improved QoL for NPC patients(9). Many other studies have confirmed these results(10-13).

Combined with the above information and based on a previous study, the present study aimed to investigate the relationships between parotid gland dysfunction and radiation dosimetry, xerostomia and QoL in NPC patients with different units after radiotherapy. This study was undertaken in order to demonstrate the superiority of IMRT compared with 3D-CRT in reducing side effects and protecting critical glands.

\section{Methods}

\section{Study patients}

From January 2019 to December 2019, a total of 80 patients were enrolled and underwent either 3D-CRT or IMRT combined with cisplatin chemotherapy at the First Hospital of Shanxi Medical University (40 patients at each), and had sufficient clinical follow-up. Eligibility criteria included: 1) NPC without distant metastasis; 2) age > 25 years; exclusion criteria included: 1) known mental or neurological diseases and history; 2) patients with cerebrovascular disease or white matter disease; 3) patients with intracranial 
invasion, intracranial primary tumor or metastasis; 4) patients with serious systemic diseases (such as hypertension, diabetes etc.).

All participants or guardians gave written informed consent and the study was approved by the institutional ethical review board.

\section{D-CRT protocol}

Of the enrolled patients (80), 40 were randomly assigned to be treated with conventional 3D-CRT (i.e., using 3D-CRT with no attempt to spare the salivary glands). At the NPC primary site, the total dose was 7000 cGy; at the lymph node and drainage area and primary tumor electively treated region, the total dose was $4400 \mathrm{cGy}$ in 2-Gy fractions. Patients were generally treated with concomitant cisplatin chemoradiation.

\section{IMRT protocol}

The aim for all patients who underwent IMRT was to spare the parotid tissue without compromising the radiotherapy efficacy. Compared with the ipsilateral gland, sparing of the contralateral gland was preferred; meanwhile, the average dose of the parotid gland was as low as possible, preferably under 26 Gy. When the ipsilateral parotid gland could not be spared (e.g., positive ipsilateral Level II nodes, primary site close to the parotid gland), the contralateral gland needed be spared as far as possible.

The parameters of IMRT were as follows: the total dose of the NPC primary site was $7040 \mathrm{cGy}$, and the doses of lymph node(s), lymph drainage area, primary site electively treated region and low-dose effectively treated region were 6720 cGy, 6400 cGy, 6080 cGy and 5440 cGy, respectively. In addition, as for the 3D-CRT protocol, patients treated with IMRT were all treated with simultaneous cisplatin chemoradiation.

\section{Xerostomia and QoL assessment}

The xerostomia and QoL were evaluated before and after 6 months of radiotherapy (both 3D-CRT and IMRT) in patients with NPC. During the study, agents stimulating or protecting the salivary glands were not allowed.

A xerostomia questionnaire (XQ) was designed and used to estimate the degree of xerostomia based on previous literature $(14,15)$. Details of the $X Q$ are listed in Supplementary Table S1. There are 8 items, and patients rated each item on a scale from 0 to 10 . The scores for each item were added together, and higher final summary scores corresponded to more severe xerostomia.

Patients also completed a QoL questionnaire to assess the toxicity of $\operatorname{RT}(15,16)$. Four relevant domains were identified in the QoL questionnaire: eating (6 items), communication (4 items), pain (4 items) and emotion (6 items). Details of the QoL questionnaire are listed in Supplementary Table S2. Scores from all domains were added together and the summary scores were recorded. Higher scores indicated greater toxicity. 


\section{Single photon emission computed tomography (SPECT)}

SPECT was performed on all patients (both 3D-CRT and IMRT) before and at 6 months after RT to estimate the function of the parotid glands. The developer $99 \mathrm{mTcO} 4$ was infused via an elbow vein in a pellet manner, and dynamic collection was performed immediately. The data were collected at 1 frame/30 s for a total of 60 frames. After $20 \mathrm{~min}$ of injection, oral vitamin C (200 mg) was given to stimulate the glands and the data were collected again. Finally, the images were processed and analyzed.

The main indexes of SPECT were the uptake rate, secretory rate and the uptake and excretion function curve of the bilateral parotid glands. Among them, the uptake index ( $\mathrm{UI})$ and excretion index (EI) were used to evaluate the function of the parotid glands.

The formulas for UI and El were as follows:

$$
\begin{aligned}
& U I=\frac{\text { high peak }- \text { backgroud }}{\text { backgroud }} \\
& E I=\frac{\text { (acid point value }- \text { acid administration after } 6 \mathrm{~min} \text { ) }}{\text { acid point value }- \text { backgroud }}
\end{aligned}
$$

Based on the degree of reduction in functionality, the parotid gland function injury was divided into four grades(17): grade I: no obvious injury, reduction in the uptake and excretion functions $\leq 20 \%$; grade II: mild injury, reduction in the uptake and excretion functions $>20 \%$ and $\leq 40 \%$; grade III: moderate injury, reduction in the uptake and excretion functions $>40 \%$ and $\leq 60 \%$; grade IV: severe injury, reduction in the uptake and excretion functions $>60 \%$ and $\leq 80 \%$; grade V: more severe injury, decrease in the uptake and excretion functions $>80 \%$.

\section{Results}

\section{Patient-reported xerostomia and QoL}

The XQ and QoL summary scores are given in Table 1. These show that, before RT, the baseline summary scores did not differ between the two groups, and there were no significant differences in XQ and QoL scores $(P>0.05)$. After 6 months of RT, the summary scores increased in both groups, indicating increased dryness. The mean XQ scores of the 3D-CRT and IMRT groups were $55.55 \pm 19.73$ and $42.08 \pm$ 12.95, respectively. Lower scores were reported for the IMRT group compared to those of the patients treated with 3D-CRT, which meant that more severe xerostomia was experienced in the latter group at 6 months post-RT. For the QoL assessment, the mean scores of the IMRT and 3D-CRT groups were $23.25 \pm$ 6.19 and $41.63 \pm 15.01$, respectively, with the higher scores of the 3D-CRT group indicating greater toxicity than for the IMRT group. The trends in both assessments were found to be consistent. 
Table 1

$\mathrm{XQ}$ and QoL scores before and 6 months after RT

\begin{tabular}{|lllll|}
\hline \multicolumn{3}{|c|}{ XQ scores } & \multicolumn{3}{c|}{ QoL scores } \\
\cline { 2 - 5 } & Before RT & $\mathbf{6}$ months & Before RT & 6 months \\
\hline IMRT & $9.83 \pm 4.22$ & $42.08 \pm 12.95$ & $26.58 \pm 8.57$ & $23.25 \pm 6.19$ \\
\hline 3D-CRT & $9.40 \pm 4.55$ & $55.55 \pm 19.73$ & $25.75 \pm 9.57$ & $41.63 \pm 15.01$ \\
\hline For the XQ scores, higher scores signify more severe xerostomia. \\
\hline \multicolumn{2}{|l}{ For the QoL scores, higher scores signify a better QoL. } \\
\hline \multicolumn{2}{|l}{ There was a significant difference between the two groups at 6 months post-RT $(P<0.05)$} \\
\hline
\end{tabular}

An estimation of the relationships between the XQ and QoL scores of the IMRT group is detailed in Fig. 1. It revealed that there was no statistically significant correlation between the two indexes before RT ( $p>$ $0.05)$. On the contrary, a statistically significant linear correlation was found with correlation coefficients (r) of 0.84 and 0.82 , respectively, at 6 months after RT $(p=0.01)$. Higher QoL scores corresponded to higher XQ scores, which meant that the higher QoL scores were accompanied by severe XQ scores to some extent.

\section{Parotid gland dose and function}

The mean dose administered to the parotid glands in the IMRT group was $19.66 \pm 3.2 \mathrm{~Gy}$, which was significantly lower than that for the 3D-CRT group (Table 2).

Table 2

Parotid gland dose, Ul and El before and 6 months after RT

\begin{tabular}{|llllll|}
\hline & Dose & UI & & El & \\
\cline { 2 - 6 } & Post-RT & Before RT & $\mathbf{6}$ months & Before RT & 6 months \\
\hline IMRT & $19.42 \pm 5.62$ & $7.26 \pm 4.31$ & $4.76 \pm 2.20$ & $0.66 \pm 0.30$ & $0.51 \pm 0.19$ \\
\hline 3D-CRT & $45.82 \pm 28.31$ & $7.68 \pm 3.82$ & $3.50 \pm 1.43$ & $0.63 \pm 0.25$ & $0.36 \pm 0.20$ \\
\hline UI, uptake index; El, excretion index. & & & \\
\hline
\end{tabular}

The $\mathrm{UI}$ and El results are also given in Table 2. Before RT, there was no significant difference in these parameters between patients who were to receive the two RT protocols $(P>0.05)$. After 6 months of RT, the mean $\mathrm{UI}$ and El values decreased by $34.41 \%$ and $22.66 \%$ in the IMRT group and by $54.4 \%$ and $41.9 \%$ in the 3D-CRT group, respectively, which meant that the function of parotid glands declined to a greater extent in the latter group, and there was a statistically significant difference $(P<0.05)$ between the IMRT 
and 3D-CRT groups. This result indicated that IMRT was associated with a better protective effect on the parotid glands.

\section{Correlation between XQ and function of the parotid glands}

An estimation of the relationships between the XQ scores and the functioning of the parotid glands for the two groups is detailed in Fig. 2. The $r^{2}$ values were 0.82 and 0.87 for the 3D-CRT and IMRT groups, respectively. There was a good correlation between $X Q$ and parotid gland function in both groups, which showed that better gland function corresponded with lower XQ scores. The IMRT group had lower xerostomia scores and better parotid gland function than the 3D-CRT group, which may be due to the sparing of the parotid glands.

\section{Discussion}

Radiotherapy is the main treatment for NPC. Because of the special anatomical position of the nasopharynx, the irregular target area shape and the adjacent critical organs, the damage rate of normal tissues is greatly increased if conventional 3D-CRT is used. IMRT is a new radiotherapy technique that can deliver different doses to different tissues to meet clinical requirements. In short, it allows the delivery of lower doses of radiation to critical normal organs, while maintaining or increasing the primary site dose, compared to 3D-CRT. It is the most commonly used radiotherapy at present that can reduce side effects, improve quality of life and protect normal tissues.

Xerostomia and QoL reduction are common and frequent side effects in NPC patients treated with RT(6). Their severity is closely related to the degree of salivary gland injury. Under normal conditions, $87-95 \%$ of the salivary gland functions are performed by the parotid and submandibular glands. However, salivary gland tissue is sensitive to radiation, and impairment of the excretion function causes the greatest reduction in salivary gland function after radiotherapy, especially in the parotid glands. Preliminary studies have shown that the degree of xerostomia after radiotherapy is positively correlated with the degree of damage and dosage of the salivary glands. Previous studies have also shown that the secretion function of the parotid glands was better protected when the average dose was less than $24 \mathrm{~Gy}$ or $26 \mathrm{~Gy}$, and would gradually improve with time $(13,18-20)$. However, if the average dose exceeded this limit, secretion from the parotid glands in most patients was very small afterward, and there was no sign of improvement over time(21). This study has shown that IMRT treatment offers great advantages in protecting parotid gland function, reducing the side effects of radiotherapy and improving the QoL of patients after radiotherapy.

Our study monitored the degree of xerostomia and QoL after RT through patient questionnaires, methods that have been widely used and confirmed to be credible in many previous surveys(22). The XQ chosen in this study was the same as one used in a larger, prospective longitudinal study(23). The University of Washington QoL instruments, which have been widely employed in other reports, were used in this study to determine the differences between the two RT treatment strategies $(22,24,25)$. In our study, the XQ and QoL scores were self-rated by the NPC patients to compare the differences between the 3D-CRT and IMRT 
treatments. Significant improvements in xerostomia and QoL at 6 months after RT were noted in the IMRT treatment group in this study. Moreover, the correlation between xerostomia and QoL was also analyzed, and the results revealed that there was some correlation between them. In both treatment groups, a more severe degree of xerostomia was accompanied by a lower QoL assessment. Similar results could be found in a study by Lin et al., who showed there was a statistically significant correlation between xerostomia and each of the four domains of QoL after parotid-sparing IMRT(26).

The severity of xerostomia is closely related to the degree of salivary gland injury. To further explore the post-RT function of the parotid glands, SPECT was used to evaluate the morphology and function of the salivary glands. It is a noninvasive, accurate, sensitive and practical quantitative analysis method that detects the degree of salivary gland dysfunction in patients with NPC after radiotherapy and has been used in many studies $(27,28)$. Münter et al. used two quantitative parameters (change in maximal uptake and change in the relative excretion rate) to determine the change in salivary gland function after RT via SPECT(28). Eleonore et al. used SPECT to evaluate the parotid gland and submandibular-sublingual salivary gland complex uptake of 99 mtechnetium pertechnetate $(99 \mathrm{mTc0} 4)(29)$. In this study, the results showed that the parotid gland function was spared better in the IMRT group than in the 3D-CRT group. In addition, we found that there was some correlation between parotid gland function and xerostomia: more severe gland function damage corresponded to more severe xerostomia.

In general, although there was heterogeneity of patients, treatments and quality of life tools, the main difference between the two groups of patients we studied and those in the above-mentioned QoL studies was the type of RT. In our study, XQ and QoL scores improved throughout the IMRT with parotid gland sparing, as well as retention of parotid gland function. In contrast, severe xerostomia tended to stabilize or even worsen over time after 3D-CRT treatment in this study. We also found statistically significant associations between xerostomia reported by patients and overall QoL 6 months after RT, and between xerostomia and the function of the parotid glands, and the trends between them suggested that efforts to spare the parotid glands through IMRT may reduce xerostomia and further improve the QoL of NPC patients.

In conclusion, this study has demonstrated that IMRT is associated with statistically significant improvements in the reduction of xerostomia and protection of parotid gland function versus 3D-CRT. Consequently, improvements in QoL were also observed in the IMRT group in the present study. These findings demonstrate that IMRT could relieve xerostomia and improve quality of life by sparing the parotid glands without compromising the target site, and support the case for assessment of QoL in relation to NPC using a parotid gland site-specific approach.

\section{Declarations}

\section{Ethics approval and consent to participate}

All participants or guardians gave written informed consent and the study was approved by the ethical review board of First Hospital of Shanxi Medical University. 


\section{Consent for publication}

Not applicable.

\section{Availability of data and material}

The data used to support the findings of this study are available from the corresponding author upon request.

\section{Competing interests}

The Authors declare that there is no conflict of interest.

\section{Funding}

This research did not receive any specific grant from funding agencies in the public, commercial, or notfor-profit sectors.

\section{Authors' contributions}

Linjun Hu was responsible for the concept and study design, and provided critical input; Xianfeng Li and Zhenzhen Wu were responsible for laboratory experiments; Xiongwei Wang and Ruijuan Meng were responsible for data collection, data analysis and interpretation; Linjun Hu and Yanlan Zhang were responsible for drafting the manuscript.

\section{Acknowledgements}

Not applicable.

\section{References}

1. Wee JTS, Ha TC, Loong SLE, Qian C-N. Is nasopharyngeal cancer really a "Cantonese cancer" $\rrbracket$. Chin J Cancer. 2010;29(5):517-26.

2. Shanmugaratnam K, Chan SH, de-The G, Goh JEH, Khor TH, Simons MJ, et al. Histopathology of nasopharyngeal carcinoma. Correlations with epidemiology, survival rates and other biological characteristics. Cancer. 1979;44.

3. Chua MLK, Wee JTS, Hui EP, Chan ATC. Nasopharyngeal carcinoma. The Lancet. 2016;387(10022):1012-24.

4. Wee JTS, Soong YL, Chua MLK. Nasopharyngeal carcinoma-past lessons and a glimpse into the future. Chinese Clinical Oncology. 2016;5(2):14.

5. Kristensen CA, Kjaer-Kristoffersen F, Sapru W, Berthelsen AK, Loft A, Specht L. Nasopharyngeal carcinoma. Treatment planning with IMRT and 3D conformal radiotherapy. Acta Oncologica. 2007;46(2):214-20. 
6. Eisbruch, Avraham. Radiotherapy: IMRT reduces xerostomia and potentially improves QoL. Nature Reviews Clinical Oncology. 2009;6(10):567.

7. Kristensen CA, Kj?R-Kristoffersen F, Sapru W, Berthelsen AK, Loft A, Specht L. Nasopharyngeal carcinoma. Treatment planning with IMRT and 3D conformal radiotherapy. Acta Oncologica. 2007;46(2):214-20.

8. Vergeer MR, Doornaert PAH, Rietveld DHF, Leemans CR, Slotman BJ, Langendijk JA. Intensitymodulated radiotherapy reduces radiation-induced morbidity and improves health-related quality of life: results of a nonrandomized prospective study using a standardized follow-up program. International Journal of Radiation Oncology Biology Physics. 2009;74(1):1-8.

9. Pow EHN, Kwong DLW, Mcmillan AS, Wong MCM, Sham JST, Leung LHT, et al. Xerostomia and quality of life after intensity-modulated radiotherapy vs. conventional radiotherapy for early-stage nasopharyngeal carcinoma: Initial report on a randomized controlled clinical trial. Int J Radiat Oncol Biol Phys. 2006;66(4):981-91.

10. Little M, Schipper M, Feng FY, Vineberg K, Cornwall C, Murdoch-Kinch C-A, et al. Reducing Xerostomia After Chemo-IMRT for Head-and-Neck Cancer: Beyond Sparing the Parotid Glands. International Journal of Radiation Oncology*Biology*Physics. 2012;83(3):1007-14.

11. Kwong D, Pow E, Sham J, McMillan A, Leung L, Leung W, et al. Intensity-modulated radiotherapy for early-stage nasopharyngeal carcinoma. Cancer. 2004;101:1584-93.

12. Hawkins PG, Lee JY, Mao Y, Li P, Green M, Worden FP, et al. Sparing all salivary glands with IMRT for head and neck cancer: Longitudinal study of patient-reported xerostomia and head-and-neck quality of life. Radiotherapy \& Oncology Journal of the European Society for Therapeutic Radiology \& Oncology. 2018;126(1).

13. Tribius S, Bergelt C. Intensity-modulated radiotherapy versus conventional and 3D conformal radiotherapy in patients with head and neck cancer: Is there a worthwhile quality of life gain? Cancer Treatment Reviews. 2011;37(7):511-9.

14. Eisbruch A, Kim HM, Terrell JE, Marsh LH, Dawson LA, Ship JA. Xerostomia and its predictors following parotid-sparing irradiation of head-and-neck cancer. International Journal of Radiation Oncology*Biology*Physics. 2001;50(3):695-704.

15. Lin A, Kim HM, Terrell JE, Dawson LA, Ship JA, Eisbruch A. Quality of life after parotid-sparing IMRT for head-and-neck cancer: A prospective longitudinal study. International Journal of Radiation Oncology*Biology*Physics. 2003;57(1):61-70.

16. Terrell JE. QUALITY OF LIFE ASSESSMENT IN HEAD AND NECK CANCER PATIENTS. Hematology/Oncology Clinics of North America. 1999;13(4):849-65.

17. Lee N, Ping X, Quivey JM, Sultanem K, Poon I, Akazawa C, et al. Intensity-modulated radiotherapy in the treatment of nasopharyngeal carcinoma: an update of the UCSF experience. International Journal of Radiation Oncology Biology Physics. 2002;53(1):12-22.

18. Li Y, Taylor JMG, Ten Haken RK, Eisbruch A. The impact of dose on parotid salivary recovery in head and neck cancer patients treated with radiation therapy. International Journal of Radiation 
Oncology*Biology*Physics. 2007;67(3):660-9.

19. Blanco A, Deasy JO, Naqa IE, Franklin GE. Dose-volume modeling of salivary function in patients with head and neck cancer receiving radiation therapy. International Journal of Radiation Oncology*Biology*Physics. 2004;60(1, Supplement):S189.

20. Eisbruch A, Ship J, Dawson L, Kim H, Bradford C, Terrell J, et al. Salivary Gland Sparing and Improved Target Irradiation by Conformal and Intensity Modulated Irradiation of Head and Neck Cancer. World journal of surgery. 2003;27:832-7.

21. Eisbruch A, Foote RL, O'Sullivan B, Beitler JJ, Vikram B. Intensity-modulated radiation therapy for head and neck cancer: Emphasis on the selection and delineation of the targets. Seminars in Radiation Oncology. 2002;12(3):238-49.

22. Terrell J, Nanavati K, Esclamado R, Bishop J, Bradford C, Wolf G. Head and Neck Cancer-Specific Quality of Life: Instrument Validation. Archives of otolaryngology--head \& neck surgery. 1997;123:1125-32.

23. Eisbruch A, Kim HM, Terrell JE, Marsh LH, Dawson LA, Ship JA. Xerostomia and its predictors following parotid-sparing irradiation of head-and-neck cancer. Int J Radiat Oncol Biol Phys. 2001;50(3):695-704.

24. C. W, Scarantino, and, F., Leveque, and, et al. A phase III study on the concurrent use of oral pilocarpine to reduce hyposalivation and mucositis associated with radiation therapy in head and neck cancer patients. Final results of RTOG 97-09. International Journal of Radiation Oncology*Biology*Physics. 2001.

25. Fisher J, Scott C, Scarantino CW, Leveque FG, White RL, Rotman M, et al. Phase III quality-of-life study results: impact on patients' quality of life to reducing xerostomia after radiotherapy for headand-neck cancer-RTOG 97-09. International Journal of Radiation Oncology*Biology*Physics. 2003.

26. Lin A, Kim HM, Terrell JE, Flemming D, Dawson LA, Eisbruch A. Head and neck cancer-specific quality of life ( $\mathrm{HN}-\mathrm{QOL})$ and its correlation with xerostomia following parotid-sparing irradiation (RT) of head and neck cancer. International Journal of Radiation Oncology*Biology*Physics. 2002;54(2, Supplement):167.

27. Bussels B, Maes A, Flamen P, Lambin P, Erven $K$, Hermans R, et al. Dose-response relationships within the parotid gland after radiotherapy for head and neck cancer. Radiotherapy and Oncology. 2004;73(3):297-306.

28. Münter MW, Karger CP, Hoffner SG, Hof H, Thilmann C, Rudat V, et al. Evaluation of salivary gland function after treatment of head-and-neck tumors with intensity-modulated radiotherapy by quantitative pertechnetate scintigraphy. International Journal of Radiation Oncology*Biology*Physics. 2004;58(1):175-84.

29. Eleonore, Huang, Ingo, Steffen, Mathias, Lukas, et al. Normal Values for Parotid Gland and Submandibular-Sublingual Salivary Gland Complex Uptake of 99mTechnetium Pertechnetate using SPECT in Mice with Respect to Age, Sex, and Circadian Rhythm. Nuklearmedizin Nuclear Medicine. 2019. 

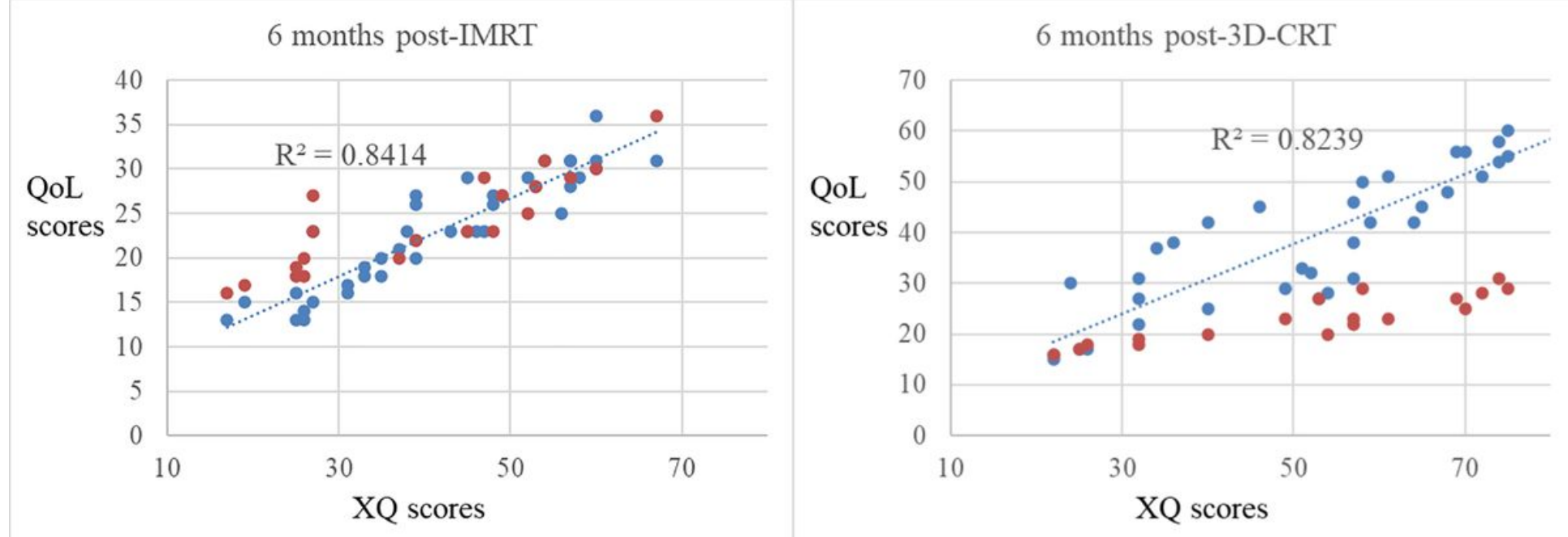

Figure 1

Correlation between XQ scores and QoL scores at 6 months post-RT. There was some correlation between the two groups at 6 months post-RT ( $r 2=0.74)$.

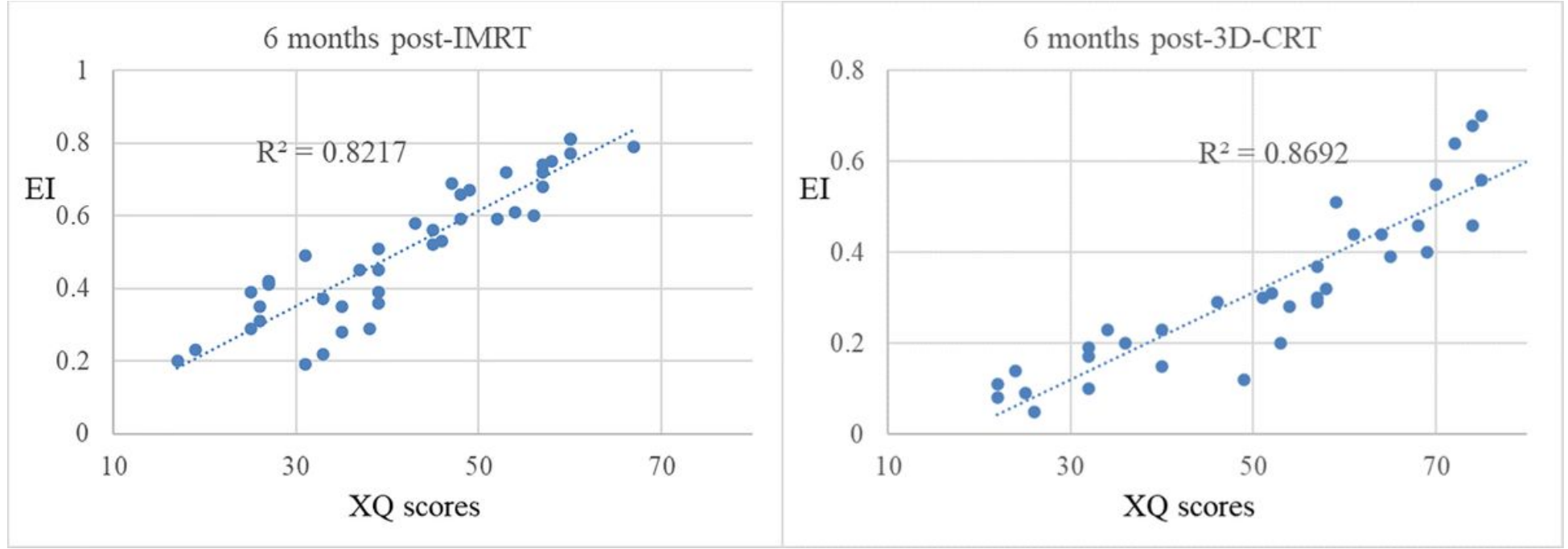

Figure 2

Correlation between XQ scores and reduction of the parotid gland El at 6 months post-RT. There was some correlation between the two groups at 6 months post-RT $(r 2=0.83)$.

\section{Supplementary Files}

This is a list of supplementary files associated with this preprint. Click to download.

- TableS1.docx 
- Tables2.docx

Page 13/13 